http://dx.doi.org/10.35381/r.k.v5i1.792

\title{
Flipped Classroom y el valor de la responsabilidad
}

\section{Flipped Classroom and the value of responsibility}

\author{
Lilia Isaurina Álvarez-Ávila \\ lilia.alvarez@psg.ucacue.edu.ec \\ Universidad Católica de Cuenca, Azogues \\ Ecuador \\ https://orcid.org/0000-0001-8315-925X \\ Darwin Gabriel Garcia-Herrera \\ dggarciah@ucacue.edu.ec \\ Universidad Católica de Cuenca, Azogues \\ Ecuador \\ https://orcid.org/0000-0001-6813-8100 \\ Nancy Marcela Cárdenas-Cordero \\ ncardenasc@ucacue.edu.ec \\ Universidad Católica de Cuenca, Azogues \\ Ecuador \\ https://orcid.org/0000-0002-6250-6504 \\ Juan Carlos Erazo-Álvarez \\ icerazo@ucacue.edu.ec \\ Universidad Católica de Cuenca, Cuenca \\ Ecuador \\ https://orcid.org/0000-0001-6480-2270
}

Recibido: 25 de abril de 2020

Revisado: 20 de mayo de 2020

Aprobado: 14 de junio de 2020

Publicado: 29 de junio de 2020 


\title{
RESUMEN
}

El objetivo de la investigación fue analizar el valor moral de la responsabilidad con respecto a la Flipped Classroom, el cumplimiento de tareas en las aulas virtuales, es decir, revisar las circunstancias que tiene los chicos en los sitios de estudio, fuera de la institución educativa, con la relación del cumplimiento responsable de sus obligaciones educativas. Se basó en el método hipotético - deductivo, con un tipo descriptivo correlacional transversal no experimental, mediante cálculo de Chi-cuadrado de Pearson. En base a los resultados obtenidos, se puede concluir con los datos de la asistencia a las clases virtual por Zoom y a los resultados de entrega o no de tareas en la plataforma Runachay, que, el grado de responsabilidad de los estudiantes como bajo, por cuanto existe una porcentaje relativamente alto de inasistencias y de incumplimiento.

Descriptores: Ética; valores morales; aprendizaje activo, método de aprendizaje. (Palabras tomadas del Tesauro UNESCO).

\begin{abstract}
The objective of the research was to analyze the moral value of responsibility with respect to the Flipped Classroom, the fulfillment of tasks in virtual classrooms, that is, to review the circumstances that children have in study sites, outside the educational institution, with the relation of the responsible fulfillment of its educational obligations. It was based on the hypothetical-deductive method, with a non-experimental transversal correlational descriptive type, using Pearson's Chi-square calculation. Based on the results obtained, it is possible to conclude with the data of the attendance to the virtual classes by Zoom and the results of the delivery or not of tasks on the Runachay platform, which, the degree of responsibility of the students as low, because there is a relatively high percentage of absences and non-compliance.
\end{abstract}

Descriptors: Ethics; moral values; activity learning; learning methods. (Words taken from the UNESCO Thesaurus). 


\section{INTRODUCCIÓN}

Tanto la educación tradicional como la educación en valores son esenciales para el desarrollo personal y ayudan a definir objetivos en la vida. Pero, mientras la primera enseña sobre el conocimiento social, científico y humanístico, la segunda brinda las herramientas necesarias para ser buenos ciudadanos. La adecuada formación y educación en valores promueve la tolerancia y la comprensión más allá de las diferencias políticas, culturales y religiosas, poniendo especial énfasis en la defensa de los derechos humanos, la protección de las minorías étnicas y los grupos más vulnerables y la conservación del medio ambiente.

Del mismo modo que se aprende matemáticas e idiomas, también se debería dar el mismo grado de importancia en aquellas lecciones que son fundamentales para vivir en armonía y el progreso social, como el respeto, la empatía, la igualdad, la solidaridad y el pensamiento crítico. Sin estos y otros principios éticos que definen al ser humano, será difícil construir un mundo mejor. A pesar de que este aspecto tan importante es considerado un eje transversal en la educación, como indica (Miniesterio de Educción del ecuador, 2016), se ha visto que en las instituciones educativas podría no estarse asumiendo debidamente, lo que puede estar repercutiendo en la conducta de los estudiantes.

La responsabilidad es un valor humano esencial, que se transforma a su vez, en una forma que, interpreta y descubre la realidad presente, además, sirve para dar el giro hacia un ciudadano del futuro, según indica (Columbié-Reyes, Columbié-Ortiz, \& BroockRomero, 2016) que existe un patrón entre los jóvenes que en cierto grado se repite en su vida estudiantil, ingresan con mucha curiosidad y expectativa de lo que va a suceder dentro de este proceso, varios encuentran lo que buscan y se sienten motivados, con algunos, sin embargo, algo puede ocurrir que le lleva a alejarse de la vida estudiantil, sin retirarse de la escuela, ocupando su tiempo otras actividades junto a sus compañeros, esta situación se la puede revertir poniendo en práctica una libertad con responsabilidad. Frente a esta situación, el docente tiene la responsabilidad de generar estrategias con las que pueda tener toda la atención de los jóvenes, de manera que, el cumplimiento de 
sus actividades autónomas con la debida responsabilidad dentro de los entornos virtuales, en este caso el aula invertida, se cumplan de acuerdo a los cronogramas propuestos. Se debe indicar que, la responsabilidad se cultiva desde tempranas edades, en la familia, como un ejemplo de las personas mayores.

Igualmente, los individuos que falten a las normas establecidas la misma sociedad es la encargada de castigarlos, así como, también lo es reconocer el cumplimiento de estas. Para (Páramo-Morales, 2017), el comportamiento humano esencialmente es cultural, siendo los valores parte de esa cultura, porque se construyen con el aporte social y el entorno. En este sentido, el aporte que se haga desde los valores, específicamente desde la responsabilidad de la persona, en primer lugar, debe tener cambios en su cultura personal para que esta cultura pueda influenciar en su comportamiento.

Al mismo tiempo, el ser humano es un ser racional, siempre dispuesto a conocer la verdad, con la educación busca conocer los fenómenos que suceden a su alrededor. Dentro de este contexto (García-Anchorena, Grimaldo Muchotrigo, \& Manzanares Medina, 2016); (Gomez, 2017) indican, la educación es un proceso inconsciente, desde el momento del nacimiento y va modelando las facultades del individuo. Los valores son una parte fundamental de la vida del hombre, en un sentido similar, necesita de la educación para convivir con la sociedad, si bien, la formación en valores se ha delegado a la educación religiosa, sin ser obligación solo de esta, los valores se desarrollan día a día, ya sea en la vida, el trabajo, la verdad, entre otros, por ende, a más de brindar conocimientos formales, desarrollar habilidades y destrezas, las instituciones educativas se ven obligadas a formar en valores.

Sin embargo, todos los centros educativos tienen diferencian según su tipo, ya que, su misión, visión y valores son generados en función de esta. En palabras de (GarcíaAnchorena, Grimaldo Muchotrigo, \& Manzanares Medina, 2016) las instituciones religiosas procuran promover desde el compromiso social y la fe, los valores como el respeto, la justicia, la solidaridad y el amor, a su vez varias políticas educativas refieren a la escuela como la encargada de enseñar valores cívicos, es decir formar ciudadanos. Desde el punto de vista sicológico, que define a los valores como creencias o 
representaciones mentales, además de ser flexibles a lo largo del tiempo, que logra un cambio a través de un proceso de resocialización.

Toda sociedad plantea, según la época que atraviesa, sus propios fines educativos, en palabras de (Torres Cañizález \& Cobo Beltrán, 2017), toda la educación se plantea prestando atención a la organización y el nivel de desarrollo de las fuerzas productivas, al igual que, tomando en cuenta la distribución de poder entre los grupos sociales, por esta razón, se han expuesto a través de los años varias instancias internacionales sus planteamientos ideológicos, en los que enuncian el desarrollo de la personalidad como el fin más importante de la educación, dando mayor prioridad a la enseñanza de valores morales, el cuidado de un planeta verde, al igual que, el descubrimiento del potencial individual en función de las necesidades de productividad.

Así mismo, existe una pérdida generalizada de valores morales dentro de la sociedad. Por estudios realizados, según (Páramo-Morales, 2017) indica, se conoce que esta situación ocurre, especialmente en Latinoamérica, entre los cuales se puede encontrar la falta de responsabilidad en las actividades delegadas dentro de la sociedad, esta percepción puede ser discutible, pero en la mayoría de casos se concluye que existe esta problemática a nivel generalizado en las instituciones educativas. Al percibir esta realidad y para afrontar esta situación, un área de atención prioritaria debería ser la educación general básica, específicamente, la básica superior, en donde, se ha podido ejecutar una propuesta mediante la aplicación de metodologías activas y el uso de la tecnología a través del aula invertida.

Actualmente, en pleno siglo XXI y con la presencia de la tecnología en cualquier lugar, se está dejando a un lado el uso de dispositivos como el proyector de diapositivas o de video, y llegando ahora a las pizarras digitales interactivas, que reúnen en un solo elemento la imagen, audio, aplicaciones, entre otros. La mayoría de herramientas recientes que se encuentran en el aula son resultado del desarrollo tecnológico, desde la perspectiva de (Sánchez-García \& Toledo-Morales, 2017) y (Chen-Hsieh, Wu, \& Marek, 2017) en la actualidad el uso en las aulas de computadores, pizarras digitales, Tablets 0 Smartphones, permiten u obligan al cambio del rol del docente así como en las formas 
de aprender de los alumnos, algunas tendencias como son el uso Bring Your Own Device (BYOD), realidad aumentada (RA) y las Flipped Classroom (FC), los que impulsan el uso de dispositivos móviles dentro del marco teórico.

En cuanto a las metodologías activas tenemos varias alternativas, (Massut-Bocklet, 2016) y (Galindo-Domínguez, 2018) describen una de ellas, y que ha ganado gran popularidad entre docentes y estudiantes, son las aulas invertidas, también conocidas como "Flipped Classroom". Esta tecnología generará un necesario cambio en el rol docente para ajustarse a nuevas experiencias, esta metodología invierte los espacios de aprendizaje, pues las clases ya no se utilizan para las explicaciones del profesor, sino para la discusión, análisis y ejecución de tareas, además de, potenciar determinados procesos de adquisición y práctica de conocimientos en base al aprendizaje obtenido en la comodidad de su casa por el estudiante, a través de, la observación de videos educativos enviados por profesor a las diferentes plataformas utilizando principalmente vídeos, presentaciones o podcasts, para poder emplear el valioso tiempo de clase para facilitar y solucionar interrogantes de los alumnos.

Dicho de otra manera, para trabajar eficientemente con TIC, (Sánchez-García \& ToledoMorales, 2017) señala que, se requiere de la adaptación de la formación del docente desde el inicio de su preparación, además de, una formación continua, y sobre todo, tener la apertura a nuevas ideas y deseos de participar en el descubrimiento de los usos de lo novedoso de la tecnología. Así mismo, deben estar claros los nuevos roles que se dan al momento de generar nuevos contenidos y el proceso de evaluación con la utilización de estos medios.

En el caso que se desarrolla, se utiliza la plataforma de la institución, Unidad Educativa "Alicia Loza Meneses", en este caso es Runachay, como indica en la página web de Grupo (Grupo EL Comercio, 2019) y de (Runachay, 2020), es una plataforma de gestión educativa y administrativa, para escuelas y colegios, cabe recalcar que, Runachay traducido al español quiere decir Educando Personas, su principal finalidad es automatizar los principales procesos de la institución educativa, una de las principales características con la cuenta justamente es efectuar una adecuada comunicación entre 
las instituciones y los padres de familia. En la actualidad cuenta con la herramienta para el control de calificaciones para padres de familia de manera instantánea, es decir, pueden visualizar al instante que el maestro actualiza las listas, además, puede realizar el proceso de matriculación en línea.

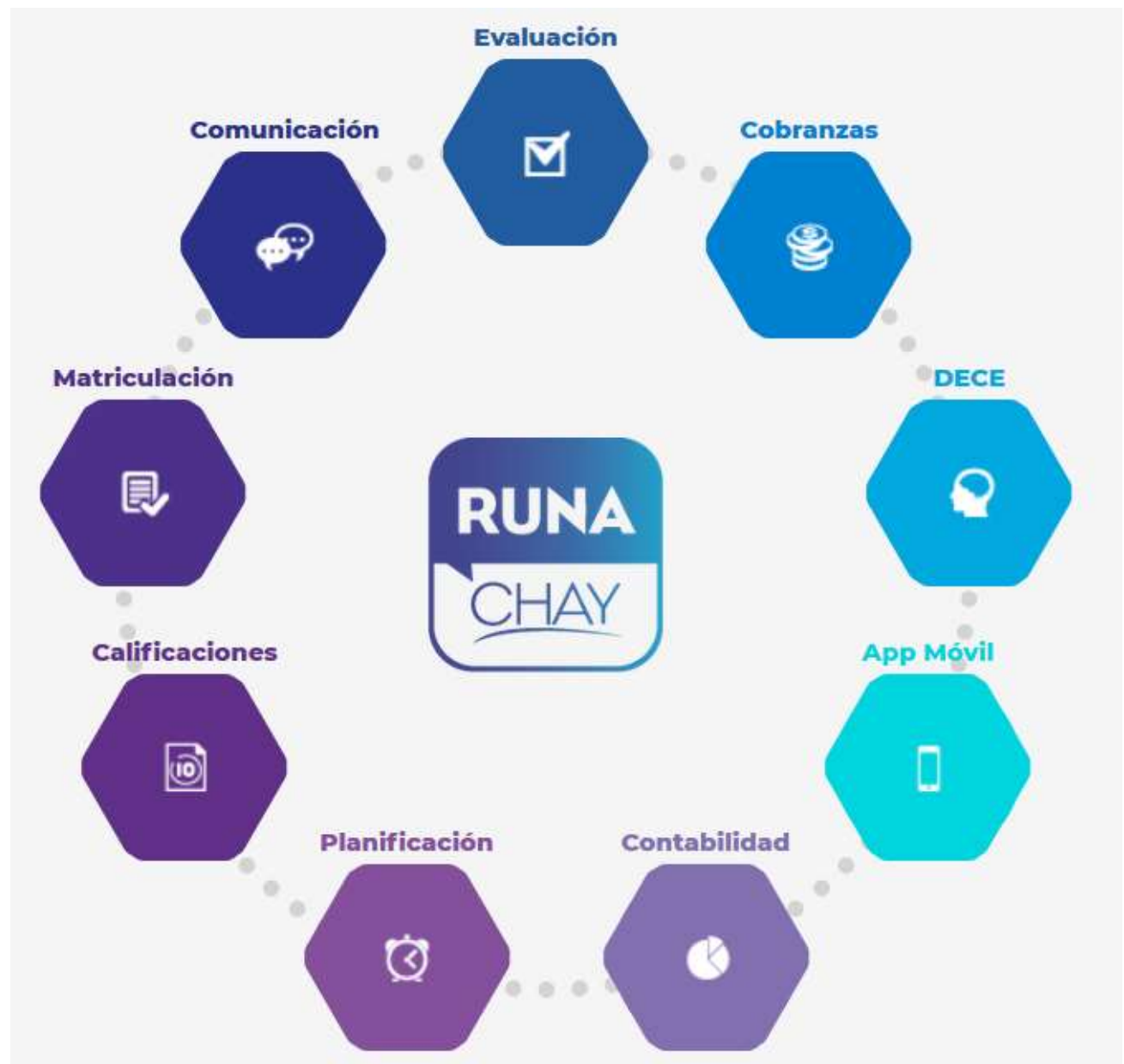

Figura 1. Módulos que posee Plataforma Runachay. Fuente. Runachay (2020)

Por otra parte, para realizar aclaraciones sobre los temas que reciben los jóvenes se utiliza clases de virtuales por medio de la plataforma zoom, como indica (Chiñas-Palacios, Vargas-Salgado, Águila-León, \& García, 2019) y (Zoom Video Communications, Inc., 2020), los docentes tienen que crear una cuenta, luego generar una reunión, y compartir el código de invitación a sus alumnos, los jóvenes a su vez, se conectan a la sesión de 
zoom mediante sus diversos dispositivos tecnológicos, para luego iniciar la clase, el docente puede compartir su pantalla para mostrar los contenidos elaborados, así mismo puede grabar la clase para luego enviarla a los alumnos. En todo momento, los alumnos pueden realizar preguntas a través de los micrófonos de sus dispositivos, se manera similar, el docente puede ver a sus alumnos en su pantalla y medir el grado de atención que tienen a la clase, en caso de ser necesario. La figura 2 muestra la pantalla de inicio de la plataforma zoom.

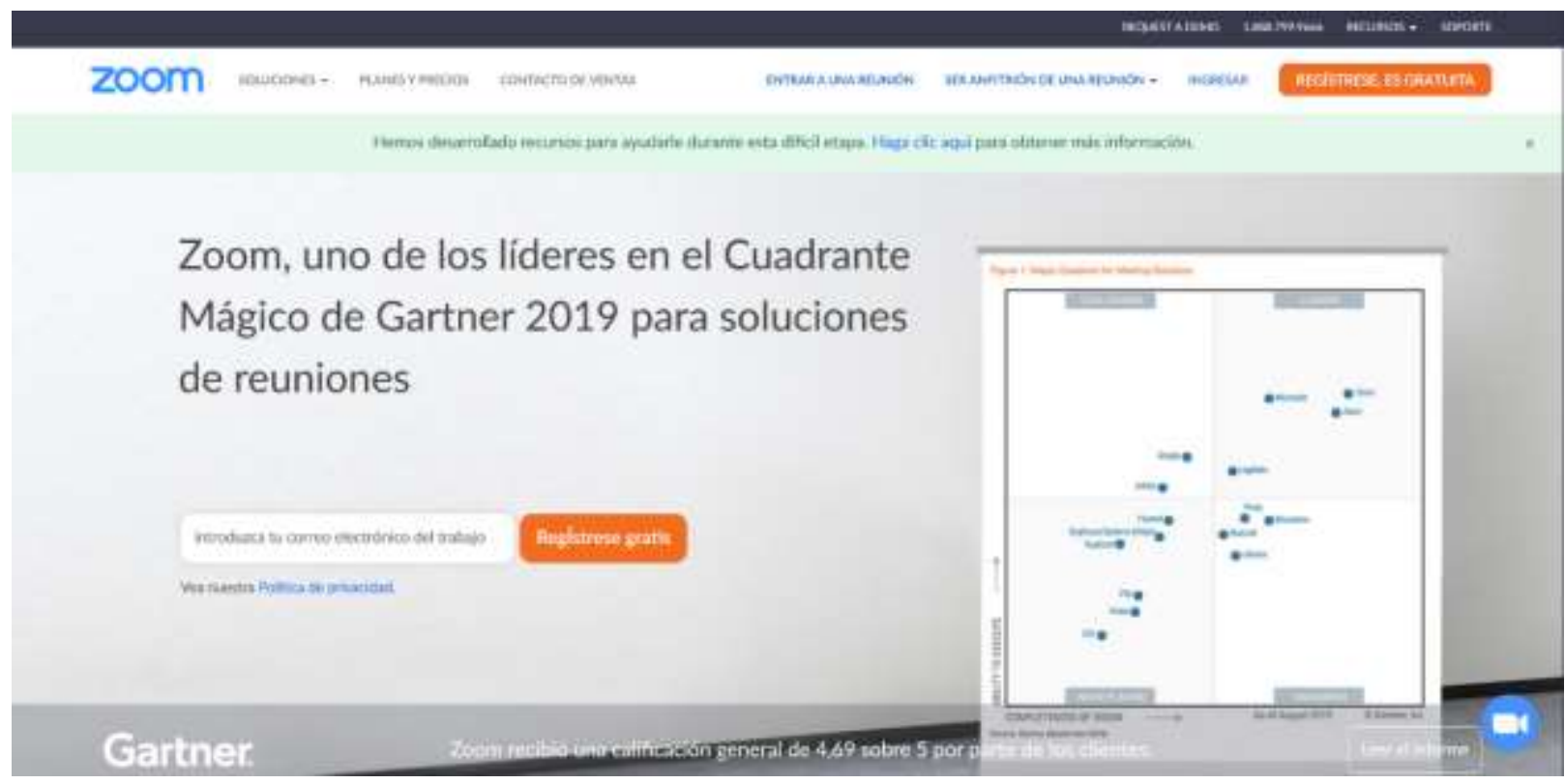

Figura 2. Pantalla inicial de Zoom. Fuente. Zoom Video Communications, Inc. (2020)

En palabras de (Blau, Weiser, \& Eshet-Alkalai, 2016), la plataforma zoom permite a los participantes mantener interacciones, al igual que verse y escucharse, bajo un entorno de educación, en el que, se ocasionan reacciones espontaneas entre los actores del proceso educativo, tanto profesor - alumnos como entre alumnos. Indica que realizó un estudio sobre el aprendizaje como se ve afectado en la videoconferencia cara - cara, unidireccional y bidireccional, igualmente, los estilos de enseñanza - aprendizaje y los rasgos de personalidad del estudiante en el logro; concluye que el logro es mayor en aprendizaje presencial que en video conferencia de una vía, también indica, que los 
aspectos del aprendizaje mostraron una notable ventaja para los participantes emocionalmente estables y los extrovertidos en comparación con los estudiantes neuróticos e introvertidos (Chirino-García \& Hernández-Corona, 2020).

La investigación presente tiene como objetivo analizar el valor moral de la responsabilidad con respecto a la Flipped Classroom, el cumplimiento de tareas en las aulas virtuales, es decir, revisar las circunstancias que tiene los chicos en los sitios de estudio, fuera de la institución educativa, con la relación del cumplimiento responsable de sus obligaciones educativas.

\section{MÉTODO}

La investigación se basó en el método hipotético - deductivo, con un tipo descriptivo correlacional transversal no experimental, mediante cálculo de Chi-cuadrado de Pearson (Paltán-Angumba, Erazo-Álvarez, \& Narváez-Zurita, 2020). La muestra fue tomada en los Octavos y Novenos de educación básica, comprendidos entre las edades de 13 y 14 años, de la Unidad Educativa Fiscomisional "Alicia Loza Meneses". La muestra se obtuvo mediante un muestreo aleatorio simple, los datos se obtuvieron mediante la aplicación de encuestas comprendida de 9 preguntas desarrolladas en la herramienta Microsoft Forms, y enviadas por medio de la plataforma Runachay de la institución.

El total de jóvenes de octavo y noveno de educación básica son 73 estudiantes, de ese total se tomó una muestra de 62 alumnos, esta muestra corresponde al resultado calculado con un $95 \%$ de nivel de confianza y un margen de error del 5\%. En la primera parte de la encuesta se colocó preguntas demográficas, de manera que, se pueda relacionar la falta del valor moral de la responsabilidad con las condiciones socioeconómicas que rodea a cada estudiante encuestado.

En la siguiente parte se colocó preguntas sobre la responsabilidad, vista desde la perspectiva del estudiante, logrando que, el mismo alumno indique la percepción propia sobre su grado de responsabilidad. Además de estos datos, se contó con el registro de tareas y asistencias a las clases virtuales, que se han emitido hasta la fecha de la toma 
de la muestra, a través de la herramienta zoom, así como la retroalimentación mediante la plataforma Runachay de la institución.

Las pruebas estadísticas se realizaron con la herramienta IBM SPSS Stadistics, con el apoyo de la Universidad Católica de Cuenca, mediante este software se realizó el análisis estadístico de las muestras obtenidas, para luego realizar las conclusiones. El instrumento para la toma de datos fue validado a través del análisis de fiabilidad con una alfa de Cronbach de 0.701.

\section{RESULTADOS}

Para el análisis de los resultados se consideró un coeficiente de confianza del 95\%, y un error de $5 \%$, se planteó la hipótesis de que en la actualidad la pérdida de valor morales se ha acentuado, en especial, la perdida de responsabilidad, que es el objetivo de esta investigación. Para el análisis de las variables se efectuó a través de tablas cruzadas y la prueba del Chi cuadrado, con estas herramientas de va a validar o negar la hipótesis planteada.

En la figura 3 se registró los resultados de la asistencia de los alumnos a las clases virtuales a través de zoom, se evidenció que un alto porcentaje de la población estudiantil no ingresa desde su casa o lugar de estudio, estos datos indicaron un alto grado de irresponsabilidad en cuanto a sus clases, estos datos reflejaron sobre otro indicador que el cumplimiento de tareas en el aula virtual, que, en similar porcentaje, no se cumple las tareas propuestas por el docente. 


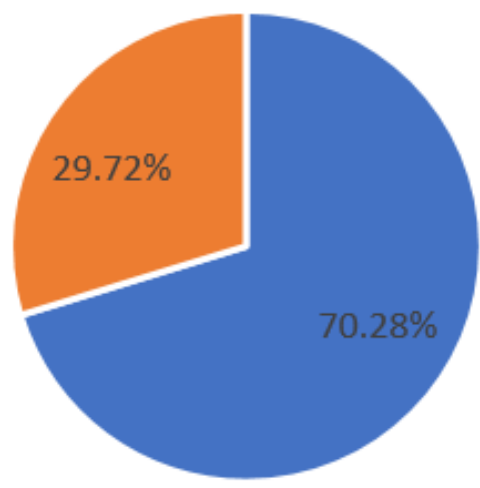

Figura 3. Registro de asistencia promedio a clases virtuales. Fuente. Elaboración propia

La tabla 1 muestra el cruce de las variables herramientas tecnológicas con el cumplimiento de tareas en tiempo y forma adecuada, donde, demostró que la mayoría de jóvenes poseen ya sea computador portátil o computador de escritorio, adicional, se tomó la hipótesis nula, que las herramientas tecnológicas no están en relación con el cumplimiento de tareas a tiempo, pero, el análisis demostró que, si existe una influencia de la herramienta tecnológica que poseen los entrevistados para cumplir adecuadamente en forma y tiempo con las tareas asignadas por el maestro, es decir si tienen una mejor herramienta, pueden cumplir mejor sus tareas. 


\section{Tabla 1}

Tabla de contingencia, Herramientas tecnológicas que posee * Cumple las tareas y actividades en tiempo y de forma adecuada. Chi cuadrado.

\section{Realiza las tareas y actividades que en} encomienda en tiempo y de forma adecuada

\section{Total}

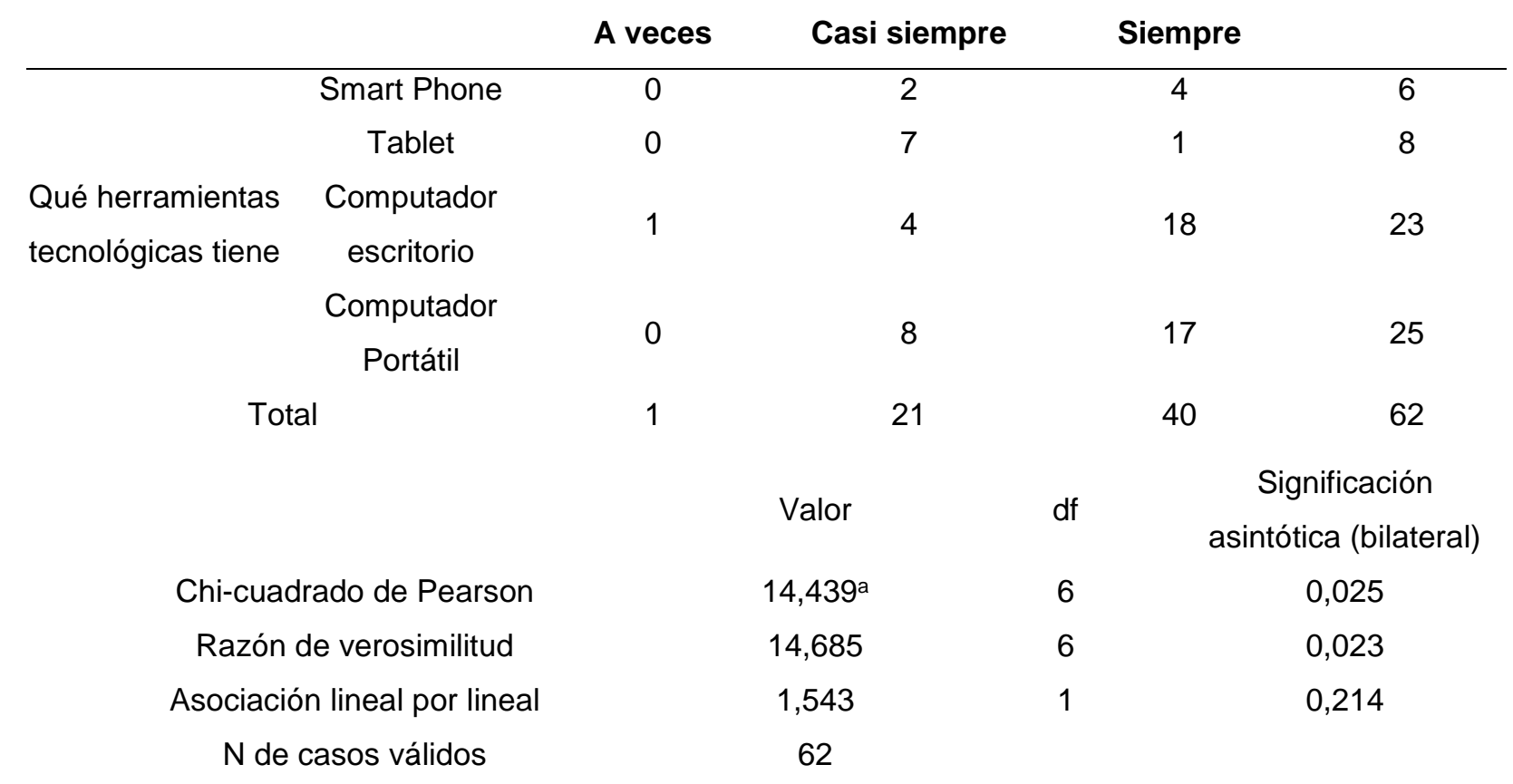

a. 7 casillas $(58,3 \%)$ han esperado un recuento menor que 5 . El recuento mínimo esperado es 10 .

La tabla 2 demostró, con el cruce de las variables autopercepción como una persona responsable y la situación laboral de los padres, que la mayoría de jóvenes tienen a sus 2 padres trabajando, de igual manera, se consideró la hipótesis nula, de esta manera, no existe relación entre la situación laboral de los padres con la autopercepción de responsabilidad, la cual fue aceptada, según el resultado del análisis del Chi cuadrado el error se consideró muy alto para afirmar una relación entre las variables. 


\section{Tabla 2}

Tabla cruzada Situación laboral de padres*Se considera una persona responsable, puntual y honesta en la entrega de las tareas y actividades. Chi Cuadrado.

\begin{tabular}{|c|c|c|c|c|c|c|}
\hline & \multicolumn{4}{|c|}{$\begin{array}{l}\text { Se considera una persona responsable, puntual y } \\
\text { honesta en la entrega de las tareas y actividades }\end{array}$} & \multirow{2}{*}{ Total } \\
\hline & & Casi nunca & A veces & $\begin{array}{c}\text { Casi } \\
\text { siempre }\end{array}$ & Siempre & \\
\hline \multirow{3}{*}{$\begin{array}{c}\text { Situación } \\
\text { laboral de } \\
\text { padres }\end{array}$} & $\begin{array}{c}\text { Sólo mamá } \\
\text { trabaja }\end{array}$ & 0 & 0 & 2 & 2 & 4 \\
\hline & $\begin{array}{l}\text { Sólo papá } \\
\text { trabaja }\end{array}$ & 1 & 1 & 11 & 11 & 24 \\
\hline & $\begin{array}{c}\text { Ambos padres } \\
\text { trabajan }\end{array}$ & 0 & 3 & 10 & 21 & 34 \\
\hline \multirow[t]{2}{*}{ Total } & & 1 & 4 & 23 & 34 & 62 \\
\hline & & \multicolumn{2}{|c|}{ Valor } & df & \multicolumn{2}{|c|}{ Significación asintótica (bilateral) } \\
\hline \multicolumn{2}{|c|}{ Chi-cuadrado de Pearson } & \multicolumn{2}{|c|}{$4,216^{a}$} & 6 & \multicolumn{2}{|c|}{0,647} \\
\hline \multicolumn{2}{|c|}{ Razón de verosimilitud } & \multicolumn{2}{|c|}{4,785} & 6 & \multicolumn{2}{|c|}{0,572} \\
\hline \multicolumn{2}{|c|}{ Asociación lineal por lineal } & \multicolumn{2}{|c|}{0,532} & 1 & \multicolumn{2}{|c|}{0,466} \\
\hline \multicolumn{2}{|c|}{$\mathrm{N}$ de casos válidos } & \multicolumn{2}{|c|}{62} & & & \\
\hline
\end{tabular}

Fuente. Elaboración propia

Otro parámetro para medir la responsabilidad de los estudiantes se analizó en la tabla 3, con este propósito se utilizó la hipótesis nula, la misma que indicó que, no existe relación entre la variable tipo de conexión y la responsabilidad de entregar las tareas y prácticas cumpliendo las instrucciones que el profesor indicó, en primera instancia se evidenció que la mayoría de estudiantes cuentan con una conexión fija de internet, además, el resultado del Chi cuadrado demostró lo contrario a la hipótesis nula, es decir, el cumplimiento adecuado de las tareas según las instrucciones del docente va en relación directa con el tipo de conexión de internet que posee, ya que el error fue considerado muy alto. 


\section{Tabla 3}

Tabla cruzada. Qué tipo de conexión tiene * Es su responsabilidad entregar las tareas y prácticas cumpliendo las instrucciones que el profesor indicó. Chi cuadrado

Es su responsabilidad entregar las tareas y

prácticas cumpliendo las instrucciones que el

profesor indicó.

\begin{tabular}{|c|c|c|c|c|c|}
\hline \multirow{3}{*}{$\begin{array}{l}\text { Qué tipo de } \\
\text { conexión tiene }\end{array}$} & \multirow{2}{*}{ No tiene } & \multirow{2}{*}{$\begin{array}{c}\text { A veces } \\
1\end{array}$} & Casi siempre & & \multirow{2}{*}{$\begin{array}{c}\text { Total } \\
4\end{array}$} \\
\hline & & & 3 & 0 & \\
\hline & Plan celular & 0 & 1 & 2 & 3 \\
\hline & Fijo & 2 & 12 & 41 & 55 \\
\hline \multirow{2}{*}{\multicolumn{2}{|c|}{ Total }} & 3 & 16 & 43 & 62 \\
\hline & & Valor & $\mathrm{df}$ & \multicolumn{2}{|c|}{ Significación asintótica (bilateral } \\
\hline \multicolumn{2}{|c|}{ Chi-cuadrado de Pearson } & $10,817^{a}$ & 4 & \multicolumn{2}{|c|}{0,029} \\
\hline \multicolumn{2}{|c|}{ Razón de verosimilitud } & 10,786 & 4 & \multicolumn{2}{|c|}{0,029} \\
\hline \multicolumn{2}{|c|}{ Asociación lineal por lineal } & 8,924 & 1 & \multicolumn{2}{|c|}{0,003} \\
\hline \multicolumn{2}{|c|}{$\mathrm{N}$ de casos válidos } & 62 & & & \\
\hline
\end{tabular}

a. 7 casillas $(77,8 \%)$ han esperado un recuento menor que 5 . El recuento mínimo esperado es, 15 .

Fuente. Elaboración propia

Entre las preguntas se incluyó sobre la responsabilidad y colaboración en los trabajos en equipo, en este punto se obtuvo un alto porcentaje de cumplimiento, más ahora que es una temporada de cuarentena, los estudiantes no pueden realizar reuniones físicamente, sino que, deben buscar medios virtuales para lograr reunirse, este análisis dejó evidencia que no influye el lugar donde se encuentren o el medio que utilicen para lograr cumplir con los trabajos en grupo asignados por el docente.

Un análisis adicional que se realizó es la asistencia a las clases virtuales, mediante la herramienta Zoom, que va en relación con el equipo tecnológico con que cuentan en casa, se evidenció que existe un 30\% aproximadamente de los jóvenes que no asisten normalmente a sus clases; de similar forma, la plataforma con la que trabaja la Institución 
Educativa Alicia Loza, Runachay en este caso, mostró que de similar manera existe un porcentaje similar que no entrega sus tareas virtuales o lo hace de manera tardía.

La educación en valores siempre ha sido una prioridad en el ámbito educativo, en especial si se trata de instituciones religiosas, de similar forma, como indica (GonzalezAlonso \& Guzón-Nestar, 2018) y (Alcocer-Aparicio, Rodriguez-Morales, \& ArangoGonzález, 2016), los valores permiten formar personas integras, debido a que los valores están en todos los ámbitos de la sociedad, estos le ayudarán a resolver sus problemas, a tomar decisiones, lograr tomar decisiones y alcanzar resultados diferentes, de manera especial si, se mide realizan sus actividades con la responsabilidad que cada actividad lo requiere.

En una sociedad multicultural la educación en valores cobra trascendencia, debido a que, se producen otros procesos, como, la interculturalidad, forman una concepción humanística y ética. La diversidad manifiesta características de grupos y sociedades de conforman la humanidad, en palabras de (Bustamante-Alfonso, Macías-Quintana, \& Araujo-Sosa, 2018), los proyectos educativos, se enfocan en el desarrollo de las potencialidades, en función de los valores, logran transformar en medio para un desarrollo humano sostenible, incluyendo el conocimiento de las características de cada estudiante, grupo étnico y nacionalidad.

El uso de la tecnología como herramienta educativa es una tendencia actual, sin embargo, no se puede aplicar sin antes realizar una planificación entre las actividades en casa y en el aula. Para (Akçayır \& Akçayır, 2018) y (Fidalgo-Blanco, Sein-Echaluce, \& García-Peñalvo, 2020), las principales ventajas del uso del aula invertida, Flipped Classroom, que indica se basan en, los resultados alcanzados en los exámenes tradicionales mejoran así como el trabajo en equipo, las metodologías activas incrementan sus indicadores en trabajo grupal como individual, aprovecha de manera eficiente el uso de la WEB 2.0 donde los alumnos donde todos los actores son creadores y consumidores de contenidos educativos (Peche-Cruz \& Giraldo-Supo, 2019). 


\section{PROPUESTA}

Los valores morales siempre has estado presentes dentro de una sociedad, sin embargo, la enseñanza ha estado delegada a las Instituciones Educativas y el desarrollo a las mismas personas, en las tablas 4 y 5 se muestra una propuesta para generar un cambio en la actitud frente a la responsabilidad y el uso de plataformas virtuales.

\section{Tabla 4}

Propuesta para mejorar la responsabilidad y el uso de plataformas virtuales

\section{Acción}

Seminario a estudiantes, sobre el valor de la responsabilidad

Capacitación a docentes en el uso de plataformas virtuales

Capacitación a padres de familia, uso de plataformas educativas
Responsable

Unidad Educativa, profesional experto

Unidad Educativa

Unidad Educativa

\section{Periodo}

Una vez a inicio de año y una vez a mitad de año, 4 horas cada sesión

Dos horas semanales

Una vez a inicio de año y una vez a mitad de año, 2 horas cada sesión

Fuente: Elaboración propia

\section{Tabla 5}

Planificación de Capacitaciones y Seminario sobre la Responsabilidad

\section{Capacitación seminario}

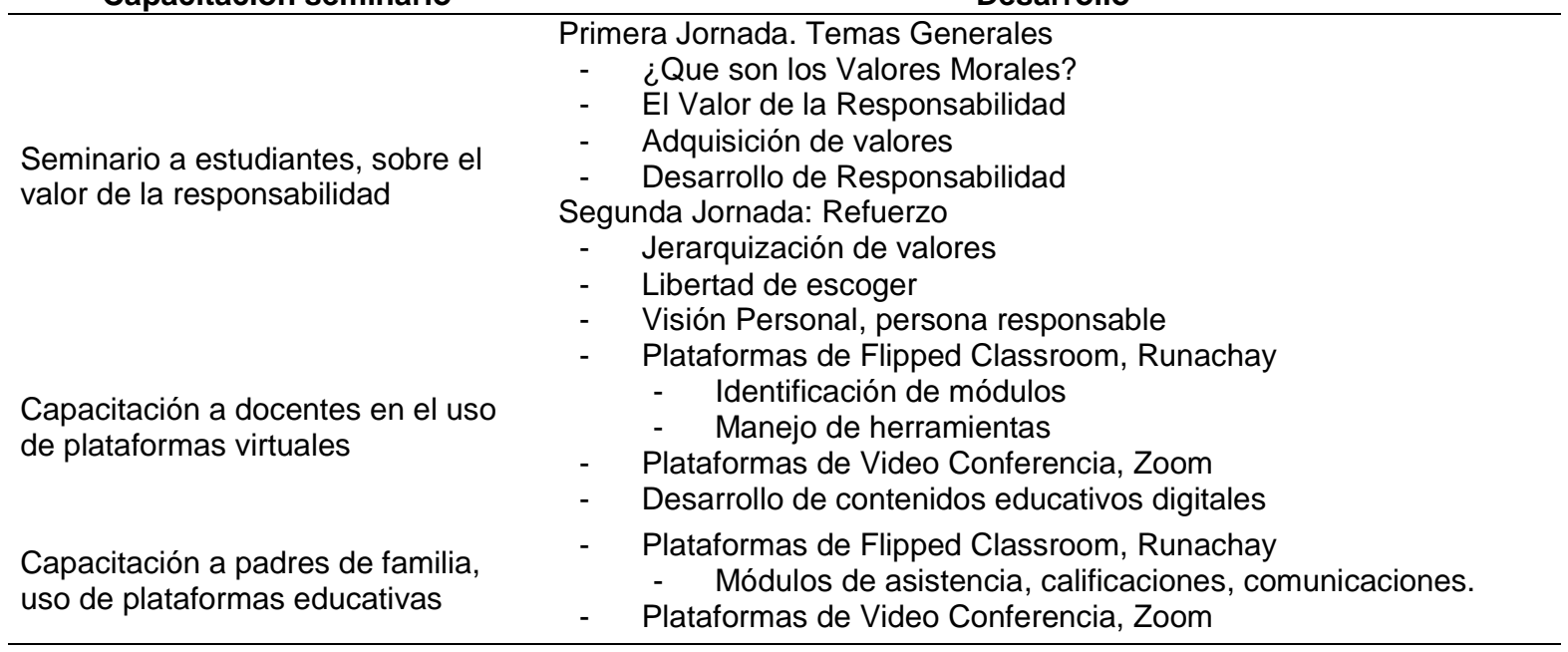

Fuente: Elaboración propia 
A la par de las capacitaciones y seminarios, para el cumplimiento de las tareas y obligaciones académicas, como sugerencia hacia los padres de familia o representantes sería mejorar las herramientas tecnológicas, por cuanto, en la nueva modalidad de estudio es muy importante tanto el equipo tecnológico, así como, la conexión a Internet con que cuente en su domicilio o lugar de estudio fuera de la escuela.

\section{CONCLUSIONES}

La percepción de la perdida de los valores morales en la sociedad se ha venido acentuando en mayor grado, en especial el valor de la responsabilidad, si bien, si medimos en base al cumplimiento de tareas, de manera fría, sin revisar el entorno en el que se encuentran los alumnos, se puede concluir que a los chicos les falta cumplir responsablemente sus actividades, pero en el análisis que se realizó en el presente estudio nos muestra que las circunstancias en la que vive o que le rodean influye; en base a los resultados obtenidos, se puede concluir con los datos de la asistencia a las clases virtual por Zoom y a los resultados de entrega o no de tareas en la plataforma Runachay, que, el grado de responsabilidad de los estudiantes como bajo, por cuanto existe una porcentaje relativamente alto de inasistencias y de incumplimiento.

Por otro lado, con los datos obtenidos a través de la encuesta realizada a los estudiantes, se evidencia que, no cumplir con las actividades indicadas por el docente está en relación directa con el tipo de conexión de Internet que posee, el cumplimiento de las tareas a tiempo tiene una relación con herramientas tecnológicas que cuenta, ya sea Smart Phone, Tablet o Computador, adicional a esto, el entorno familiar en que se desarrolla, ya sea con sus dos padres, o solo con uno de ellos no influye o no tiene relación en el grado de responsabilidad en especial en la autopercepción persona responsable que tiene de sí mismo.

Para un mejoramiento en el valor de la responsabilidad, se propone realizar seminarios con los jóvenes sobre la responsabilidad, donde, los estudiantes conocerán más sobre valores morales, en especial de la responsabilidad, lo que contribuirá con su formación humana. Si bien, las muestras y el análisis muestran que la falta de responsabilidad, en 
cuanto a tares estudiantiles en tiempo de pandemia Covid 19, es debida a factores tecnológicos, con el aporte de las capacitaciones que se propone se logra que conozca sobre la responsabilidad y la pueda aplicar en todas las actividades de su vida.

En el tiempo presente, las adaptaciones que se generan en un futuro y la necesidad del cambio, generan la toma de medidas que involucran tecnología, tanto en la vida diaria como en la educación, por lo que, se debe preparar y capacitar a las personas encargadas de la educación, en este caso a maestros y padres, por lo que con capacitaciones de acuerdo al rol que desempeña cada uno de ellos, se puede mejorar la comunicación virtual, entre personas y personas con las máquinas, de esta manera, el acompañamiento al estudiante se verá favorecido.

\section{FINANCIAMIENTO}

No monetario.

\section{AGRADECIMIENTO}

Al personal Directivo y Docente de la Unidad Educativa Fiscomisional Alicia Loza Meneses por apoyar el desarrollo de la investigación.

\section{REFERENCIAS CONSULTADAS}

Akçayır, G., \& Akçayır, M. (2018). The flipped classroom: A review of its advantages and challenges [El aula invertida: una revisión de sus ventajas y desafíos]. Computers \& Education, 334-345. https://doi.org/10.1016/j.compedu.2018.07.021

Alcocer-Aparicio, P. M., Rodriguez-Morales, A., \& Arango-González, J. L. (2016). Formacion Docente para promover valores morales en la universidad de Guayaquil [Formación Docente para promover valores morales en la universidad de Guayaquil]. Revista Universidad y Sociedad, 179-186. Obtenido de https://n9.cl/mlox 
Blau, I., Weiser, O., \& Eshet-Alkalai, Y. (15-18 de Junio de 2016). Face-to-face versus one-way and two-way videoconferencing: How medium naturalness and personality traits influence achievement and perceived learning? [Face-to-face versus one-way and two-way videoconferencing: How medium naturalness and personality trai]. 2016 11th Iberian Conference on Information Systems and Technologies (CISTI). doi:https://doi.org/10.1109/CISTI.2016.7521581

Bustamante-Alfonso, L. M., Macías-Quintana, A. M., \& Araujo-Sosa, R. (2018). Educación en valores morales y diversidad cultural: vínculo ineludible en la Escuela Latinoamericana de Medicina [Education in moral values and cultural diversity: an inescapable link at the Latin American School of Medicine]. Panorama Cuba $y$ Salud, 140-144. Obtenido de https://n9.cl/elv9

Chen-Hsieh, J. S., Wu, W.-C. V., \& Marek, M. W. (2017). Using the flipped classroom to enhance EFL learning [Usando el aula invertida para mejorar el aprendizaje EFL]. Computer Assisted Language Learning, 1-21. doi:https://doi.org/10.1080/09588221.2015.1111910

Chiñas-Palacios, C., Vargas-Salgado, C., Águila-León, J., \& García, E. X. (2019). Zoom y Moodle: acortando distancias entre universidades. Una experiencia entre la Universidad de Guadalajara, México y la Universidad Libre de Colombia [Zoom and Moodle: shortening distances between universities. An experience between the University of G]. Valencia: Editorial Universitat Politècnica de València. Obtenido de https://n9.cl/p7s5

Chirino-García, R., \& Hernández-Corona, J. (2020). M-learning: Estrategia para la promoción del aprendizaje electrónico móvil en instituciones de educación superior. [M-learning: Strategy for the promotion of mobile e-learning in higher education institutions]. EPISTEME KOINONIA, 3(5), 102-121. http://dx.doi.org/10.35381/e.k.v3i5.684

Columbié-Reyes, J. L., Columbié-Ortiz, F. J., \& Broock-Romero, Y. (2016). El valor responsabilidad como unidad de análisis del proceso formativo integral en estudiantes de ciencias médicas [The value of responsibility as a unit of analysis of the comprehensive training process in medical science students]. Medisan, 20(2), 248-253. Obtenido de https://n9.cl/on3d

Fidalgo-Blanco, Á., Sein-Echaluce, M. L., \& García-Peñalvo, F. J. (2020). Ventajas reales en la aplicación del método de Aula Invertida-Flipped Classroom [Real advantages in applying the Inverted Classroom-Flipped Classroom method]. Madrid: Liti. Universidad Politécnica de Madrid. Obtenido de https://n9.cl/g1tr 
Galindo-Domínguez, H. (2018). Un meta-análisis de la metodología flipped classroomen el aula de educación primaria [A meta-analysis of the flipped classro methodology in the primary education classroom]. EDUTEC. Revista electrónica de tecnología educativa(63), 73-85. doi:https://doi.org/10.21556/edutec.2018.63.983

García-Anchorena, R., Grimaldo Muchotrigo, M., \& Manzanares Medina, E. L. (2016). Jerarquia de valores entre estudiantes de secundaria de colegio religioso y colegio laico de Lima [Hierarchy of values between high school students of religious school and lay school of Lima]. Liberabit, 22(2), 230-238. Obtenido de https://n9.cl/fuvo

Gomez, E. L. (2017). Educación en Valores [Education in values]. Revista RAITES, 3(6), 69-87. Obtenido de https://n9.cl/4y9n

Gonzalez-Alonso, F., \& Guzón-Nestar, J. L. (2018). La educacion en valores: axiologia, naturaleza y derecho educativo [Values education: axiology, nature and educational law]. Ciencias Humanas, 90-120. Obtenido de https://n9.cl/ou4yz

Grupo EL Comercio. (7 de 11 de 2019). Líderes [Leaders]. Obtenido de https://n9.cl/tn961

Massut-Bocklet, M. F. (2016). Estudio de la utilización de vídeos tutoriales como recurso para las clases de matemáticas en el bachillerato con "Flipped Classroom" []. Universidad de Barcelona. Obtenido de https://n9.cl/zvdvb

Miniesterio de Educción del ecuador. (2016). Actualización y reforzamiento curricular de la educación general básica [Updating and reinforcing the curriculum of basic general education]. Obtenido de https://n9.cl/sbgbx

Paltán-Angumba, A. L., Erazo-Álvarez, J. C., \& Narváez-Zurita, C. I. (2020). Centro de Costeo para prestaciones de los Hospitales de la ciudad de Cuenca - Ecuador [Costing Center for benefits of the Hospitals of the city of Cuenca - Ecuador]. Revista Arbitrada Interdisciplinaria Koinonía, 638-670. doi:http://dx.doi.org/10.35381/r.k.v5i10.708

Páramo-Morales, D. (2017). Cultura y comportamiento humano [Culture and human behavior]. Pensamiento \& Gestión(42). Obtenido de https://n9.cl/d2nwt

Peche-Cruz, H., \& Giraldo-Supo, V. (2019). El Aprendizaje Flip Learning centrado en el estudiante como generador de calidad educativa. [Student-centered Flip Learning as a generator of educational quality]. Revista Arbitrada Interdisciplinaria Koinonía, 4(8), 427-450. http://dx.doi.org/10.35381/r.k.v4i8.293

Runachay. (16 de 04 de 2020). Runachay. Obtenido de https://runachay.runacode.com/public/ 
Sánchez-García, J. M., \& Toledo-Morales, P. (2017). Tecnologías convergentes para la enseñanza: Realidad Aumentada, BYOD, Flipped Classroom [Convergent Technologies for Teaching: Augmented Reality, BYOD, Flipped Classroom]. Revista de Educación a Distancia, 1-15. Obtenido de https://n9.cl/41ex

Torres Cañizález, P. C., \& Cobo Beltrán, J. K. (2017). Tecnología educativa y su papel en el logro de los fines de la educación [Educational technology and its role in achieving the ends of education]. Educere, 21(68), 31-40. Obtenido de https://n9.cl/nrcdx

Zoom Video Communications, Inc. (16 de 04 de 2020). Zoom. Obtenido de https://zoom.us/ 